\title{
Mixed Contiguous and Aggregated Spectrum Allocation Algorithm for CR based TD-LTE System
}

\author{
Xiaoyan Liu, Qingyi Quan, Peng Lei, Wenbin Guo, Wenbo Wang \\ WSPN Lab. Key Laboratory of Universal Wireless Communication, Ministry of Education, School of Information and \\ Communication Engineering, Beijing University of Posts and Telecommunications, China \\ Email: jaunt299@gmail.com
}

Received July, 2013

\begin{abstract}
Cognitive Radio (CR) is a promising technology to increase the efficiency of spectrum by opportunistic access. However, in many cases the sensed spectrum is so broken that it cannot be utilized sufficiently with traditional continuous spectrum allocation strategy. Spectrum aggregation can make full use of narrow spectrum fragments while the overhead it brings may reduce the overall performance of the system. This paper proposes a Mixed Contiguous and Aggregated Spectrum Allocation (MixCASA) algorithm for CR based TD-LTE Networks. This algorithm combines contiguous with discontiguous spectrum allocation together in order to guarantee the feasibility and efficiency of system. Simulation results show that the MixCASA algorithm can increase the number of allocated channels, and reduce the overhead brought by excessive spectrum aggregation as well.
\end{abstract}

Keywords: Cognitive Radio; TD-LTE; Spectrum Allocation; Spectrum Aggregation

\section{Introduction}

As a standard for wireless communication technology, LTE offers considerably high data rate [1]. While the spectrum usage efficiency has been improved, it is necessary to increase the transmission bandwidth to achieve a higher data rate according to Shannon formula. However, spectrum is one of the main obstacles that the developing wireless communication technology must overcome. Besides, traditional spectrum allocation strategy is proved to be inefficient [2], so finding new algorithms to assign this scarce resource more rationally is urgent.

CR is a promising way to solve this problem. In CR networks, available spectrum is automatically detected to allow the access of Secondary Users (SUs) without sensible interference to Primary Users (PUs) [3]. However, available spectrum condition of CR networks has great difference compared with that of traditional wireless communication networks. Because the sensed spectrum may not be stable all the time and its character also changes. Besides, in most cases the spectrum is so fragmented that it is hard or impossible to be utilized efficiently [3]. Owning to these facts, traditional spectrum allocation protocols are not very applicable.

Spectrum aggregation is a feasible way to settle the problems mentioned above. By bonding multiple spectrum fragments together, it allows multiple contiguous or discrete blocks of spectrum to be treated as if they are one large contiguous block [4]. However, the aggregation capacity is limited to some extent and fragments that are too scattered cannot be bonded together. Moreover, aggregation also brings overhead and it has high requirement for hardware [5]. So excessive spectrum aggregation should be avoided to reduce unnecessary overhead and guarantee the spectrum efficiency at the same time.

Besides, spectrum resource is similar to computer memory resource in some aspects, for both of them may be fragmentized during the allocation, and excessive fragments handicap their performance. In this paper, we are inspired by the best-fit strategy of computer memory allocation, which is a good way to minimize fragments [6]. Best-fit memory allocation picks out the minimum one among all the resources that satisfy user's demand. And it makes the best use of resource in this way.

Considering those mentioned above, we propose a Mixed Contiguous and Aggregated Spectrum Allocation (MixCASA) algorithm. In this algorithm, spectrum or channel resource is allocated in two ways. If there is sufficiently available contiguous resource for a certain user demand, best-fit spectrum allocation strategy is implied. Otherwise spectrum aggregation is carried out to increase the total number of channels that can be allocated as many as possible.

The rest of this paper is organized as follows. In part 2 related works are illustrated. Then the system model and the MixCASA algorithm are proposed in part 3. After 
giving the simulation results and analyzing the performance in part 4, we make our conclusions in part 5.

\section{Related Work}

In order to utilize the scarce spectrum resource more effectively and efficiently, Dynamic Spectrum Allocation (DSA) has been well studied these years. Previous works such as [7] intend to increase spectrum efficiency and guarantee user's demand in a contiguous spectrum level. However, in most cases the sensed spectrum may not be that perfect and may have many narrow fragments [8], thus it is difficult to make full use of this scarce resource with traditional continuous spectrum allocation strategy.

Wireless radio technology such as Discontiguous Orthogonal Frequency Division Multiplexing (DOFDM) [9] makes spectrum aggregation possible, in which many detached spectrum holes can be joint together and then utilized as if they were a whole block [10]. It is a promising technology for its flexibility and dynamic characteristic and low interference between adjacent subchannels when allocating spectrum resource.

Spectrum aggregation works exactly this way [11]. Spectrum aggregation makes it possible to utilize more than one carrier and in this way increase the overall transmission bandwidth. Although technique has been already quite well investigated at theoretical level, their practical implementation is not immediate and also raises numerous challenges. Intuitively speaking, the aggregation capacity cannot be unlimited [12], otherwise the corresponding hardware may not satisfy its requirement and the overhead aggregation brings may handicap the overall performance of the system as well.

Aggregation Aware Spectrum Assignment (AASA) proposed in [13] is a greedy algorithm. This algorithm gives an aggregation capacity named aggregation span, which performs in the form of sliding window. It can achieve maximum access number by assigning available channels within the sliding window and then moving the window from lower to upper frequency successively. However this greedy algorithm is not very practical because every SU in this model is set to have the same bandwidth demand and it does not take overhead brought by channel aggregation into consideration.

Maximum Satisfaction Algorithm (MSA) presented in [12] considers different bandwidth requirements of SUs.
In this algorithm SU with larger bandwidth requirement can be allocated preferentially. While it only focus on maximization of the total access number of SUs' bandwidth requirements, and still neglects the load aggregation brings to system.

MixCASA algorithm we propose in this paper aims to improve spectrum efficiency, and avoid excess aggregation at the same time by combine contiguous and discrete spectrum allocation together. So it can guarantee a relatively high number of allocated channels and avoid excessive overhead brought by spectrum aggregation. Experiment results show that this algorithm can achieve a much better performance when taking both spectrum efficiency and aggregation overhead into consideration.

\section{System Model and Algorithm}

\subsection{Scenario Definition}

We assume a CR network that has $\mathrm{N}$ SUs denoted by $U_{n}, n=\{1,2, \cdots N\}$. Considering the most common wireless communication scenario, we make some assumptions as follows.

- There are enough SUs, and every channel can only be occupied by one SU or PU.

- Every SU has a bandwidth requirement $R B_{n}^{S U}$, $n=\{1,2, \cdots N\}$. And all of these requirements compose an array $R B$.

$$
R B=\left\{R B_{1}^{S U}, R B_{2}^{S U}, \cdots, R B_{N}^{S U}\right\}
$$

where $R B$ is random distributed so that it can line with the practical situation, in which SUs have various bandwidth requirements.

- The condition of spectrum remains unchanged, which means we have a stable spectrum that has already been sensed during the allocation, and the total bandwidth is $B \mathrm{MHz}$.

- The maximum aggregation capacity is $T^{\text {threshold }}$, and the width of sliding window is $T^{\text {window }}$, which is shown in Figure 1. As window moves forward, the whole spectrum will be searched.

Assume that spectrum is unitized, so we can label these channels in integer numbers. The total spectrum can be denoted by $B^{\text {total }}$. And the total available spectrum is denoted by $B^{\text {avail }}$. Every available spectrum fragment $B_{j}^{\text {avail }}$ and its length $\left|B_{j}^{\text {avail }}\right|$ can be denoted

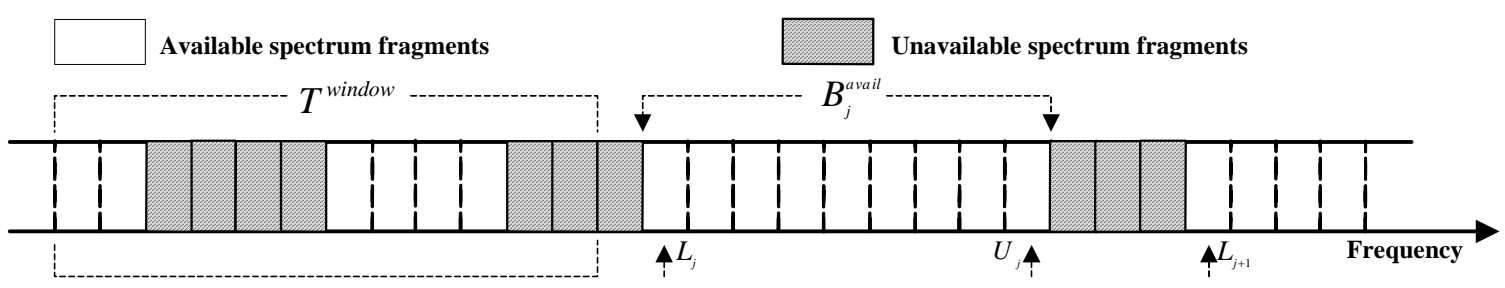

Figure 1. Fragment fragments and allocation schematic. 
as follows.

$$
\begin{gathered}
B_{j}^{\text {avail }}=\left\{L_{j}, L_{j}+1, \cdots, U_{j}\right\} \\
\left|B_{j}^{\text {avail }}\right|=U_{j}-L_{j}+1, j=\{1,2, \cdots J\}
\end{gathered}
$$

where $L_{j}$ and $U_{j}$ are the lower and upper unitized channel number of $j$ th available spectrum, which satisfy the following relationship.

$$
L_{j}<U_{j}<L_{j+1}, \quad j=\{1,2, \cdots J\}
$$

So the total unitized available channel and its length is

$$
\begin{aligned}
B^{\text {avail }} & =\bigcup_{j=1}^{J} B_{j}^{\text {avail }} \\
\left|B^{\text {avail }}\right| & =\sum_{j=1}^{J}\left|B_{j}^{\text {avail }}\right| .
\end{aligned}
$$

In this model, we assume that SU with larger bandwidth requirement has priority to be allocated firstly. Let $C$ denotes the total number of channels assigned after the whole allocation process. If $K$ SUs' requirement are accepted in all, the equation of $C$ can be denoted as follows.

$$
C=\sum_{k=1}^{K} R B_{k}^{S U}, k \in\{1,2, \cdots N\}
$$

And allocated channel numbers $C_{\text {cannot be larger }}$ than the number of total available channels. So we have

$$
C \leq\left|B^{\text {avail }}\right|
$$

And our target is to maximize $C$ and avoid excessive aggregation at the same time.

\subsection{Description of Proposed Algorithm}

In this paper, we propose a MixCASA algorithm to solve the problem described above.

The key idea of MixCASA is to allocate spectrum resource contiguously in best-fit method if there are sufficiently enough contiguous vacant channels for a SU's demand. Otherwise spectrum aggregation is applied to guarantee the number of channel that can be allocated. All SUs are allocated one by one according to their bandwidth requirements, which are sorted decreasingly. Steps of this algorithm can be simplified as follows.

1) Sort all the channel requirements of SUs decreasingly. So SU with larger demand has a priority to use the limited spectrum resource. For a certain SU, spectrum assignment can be divided into two stages.

2) During the contiguous assignment, best fit strategy is applied. And the whole sensed spectrum is searched to pick out the smallest one among all the spectrum fragments that are big enough for this SU's requirement.

3) During the discontiguous allocation, available channels within sliding window are searched and bonded together to satisfy the SU's demand. If no sufficient channels can be found after these two stages, this SU's requirement will be refused.

As mentioned above, the proposed algorithm contains contiguous channel assignment and discontiguous channel assignment. It is not hard to discover that steps of this algorithm can be simplified by setting the width of sliding window as follows.

During contiguous channel assignment stage,

$$
T^{\text {window }}=R B_{n}^{S U}, n=\{1,2, \cdots N\}
$$

And during aggregation stage, we have

$$
T^{\text {window }}=T^{\text {threshold }}
$$

The model of sliding window is shown in Figure 1. Different values of $T^{\text {window }}$ mean different allocation modes. As the sliding window moves successively, all the channels can be checked and allocated if it satisfies any one of these two modes. Detailed procedure of MixCASA is demonstrated in Algorithm 1 and Algorithm 2 .

\section{Algorithm 1: MixCASA}

Input: SUs' bandwidth requirement vector $R B$, normalized available channels $B^{\text {avail }}$, aggregation threshold $T^{\text {threshold }}$, and the width of sliding window $T^{\text {window }}$

Output: Channel state after the allocation of all SUs denoted by 0 (unallocated) and 1(allocated)

$$
\begin{aligned}
& \text { for } n=1 \text { to } N \text { do } \\
& \text { tag }=0 \\
& \text { for } j=1 \text { to }\left|B^{\text {avail }}\right| \text { do } \\
& \text { if }\left|B_{j}^{\text {avail }}\right|>0 \text { and } R B_{n}^{S U}<=\left|B_{j}^{\text {avail }}\right| \text { then } \\
& \quad S 1=j \\
& \quad / * S 1 \text { is an temporary array to store } j * / \\
& \quad t a g=1 \\
& \text { end if } \\
& \text { end for } \\
& \text { if tag }=1 \text { then } \\
& T^{\text {window }}=R B_{n}^{S U} \\
& \quad y=\text { min }(S 1) \\
& \quad / * y \text { is the location of the minimum of } S 1 \text { */ } \\
& L_{y}=L_{y}-T^{\text {window }}+1 \\
& \text { else } \\
& \text { end for } \\
& \begin{array}{l}
\text { end if } \\
\text { window }
\end{array}=T^{\text {threshold }}
\end{aligned}
$$


Algorithm 2: Spectrum Aggregation

Input: Slide-window width $T^{\text {window }}, j$ th SU's bandwidth request $R B_{n}^{S U}$, available channels $B^{\text {avail }}$

Output: Channel state after the assignment of $j$ th SU

/* p1, p2 are the start and stop location of slide window */

for $p 1=1$ to $\left|B^{\text {avail }}\right|-T^{\text {window }}$ do

$p 2=p 1+T^{\text {window }}-1$

$S 2=\operatorname{find}\left(B^{\text {avail }}(p 1: p 2)=0\right)$

/* S2 stores the location of available channels within slide window */

if length $(S 2)>=R B_{n}^{S U}$ do

$L_{p 1}=L_{p 1}+T^{\text {window }}-1$

else

$p 1=p 1+1$

end if

end for

\section{Simulation and Performance Analysis}

We configure the simulation as shown in Table 1. According to current sampling speed of analog-to-digital converters, the aggregation capacity $T^{\text {threshold }}$, or aggregation threshold we call here, is set at $40 \mathrm{MHz}$. Spectrum range is fixed at VHF and UHF within broadcasting TV band from 100 to $600 \mathrm{MHz}$. SUs' bandwidth requirements are set to range from $4 \mathrm{MHz}$ to $20 \mathrm{MHz}$. In the simulation, channel fragment number changes from 1 to 100. And when it is 1 , it means we have an unbroken spectrum, which is a theoretical condition that we take as a reference. We run these algorithms for 150 times respectively and the variables are re-generated in every run. Then we get the average values of simulation as follows.

Figure 2 shows the allocated channel number, i.e. the number of channels that can be allocated by algorithms mentioned above, including AASA from [12], MixCASA proposed in this paper and traditional contiguous spectrum allocation. Here we denote them with array $N_{A}^{\text {allo }}$, $N_{M}^{\text {allo }}$ and $N_{C}^{\text {allo }}$. AASA is a greedy algorithm, which slides the window from low to high one by one without considering the other factors. So it is a theoretical strategy but not very practical. When the number of fragments is not very big, i.e. the sensed spectrum is relatively complete, the allocated channel number of these algorithms nearly stay the same. As spectrum fragments increases, performance of oontiguous spectrum allocate strategy goes downhill quickly while the performance of MixCASA algorithm only has a slight decrease.

Figure 3 shows the aggregation number, i.e. how many times aggregation occurred during the allocation. They are denoted by array $N_{A}^{a g g}$ and $N_{M}^{a g g}$. Here we make the assumption that there is an aggregation as long as the width of slide-window is set at the maximum aggregation capability $T^{\text {threshold }}$, and channels are assigned to certain SU successfully during this assignment. Because there is no aggregation in contiguous spectrum assignment, we just present the aggregation number of AASA and MixCASA algorithm here. Compare Figure 2 with Figure 3 we can see that when the sensed spectrum is not so fragmentary, aggregation number of MixCASA is significantly reduced than that of greedy algorithm. And the number of allocated channels remains a relatively high level at the same time.

The data in Figure $\mathbf{2}$ and Figure $\mathbf{3}$ are converted in following way so that we can have a comparison more understandable and reasonable.

$$
\begin{gathered}
R_{\text {AASA }}=\frac{N_{A}^{a g g} / \max \left(N_{A}^{a g g}\right)}{N_{A}^{\text {allo }} / \max \left(N_{A}^{\text {allo }}\right)} \\
R_{\text {Mix CASA }}=\frac{N_{M}^{a g g} / \max \left(N_{M}^{a g g}\right)}{N_{M}^{\text {allo }} / \max \left(N_{M}^{\text {allo }}\right)}
\end{gathered}
$$

The results of $R_{A A S A}$ and $R_{\text {MixCASA }}$ are shown in Figure 4, which in part reflect the different overhead of aggregation when allocating sub-channels in different ways. We can see that the normalized average aggregation overhead of MixCASA is much smaller than that of AASA.

Table 1. Simulation configurations.

\begin{tabular}{ll}
\hline Parameter Name & Value \\
\hline Frequency range & $100 \mathrm{MHz}$ to $600 \mathrm{MHz}$ \\
Total available Frequency & $300 \mathrm{MHz}$ \\
Bandwidth Per Sub-carrier & $1 \mathrm{MHz}$ \\
Aggregation threshold & $40 \mathrm{MHz}$ \\
\hline
\end{tabular}

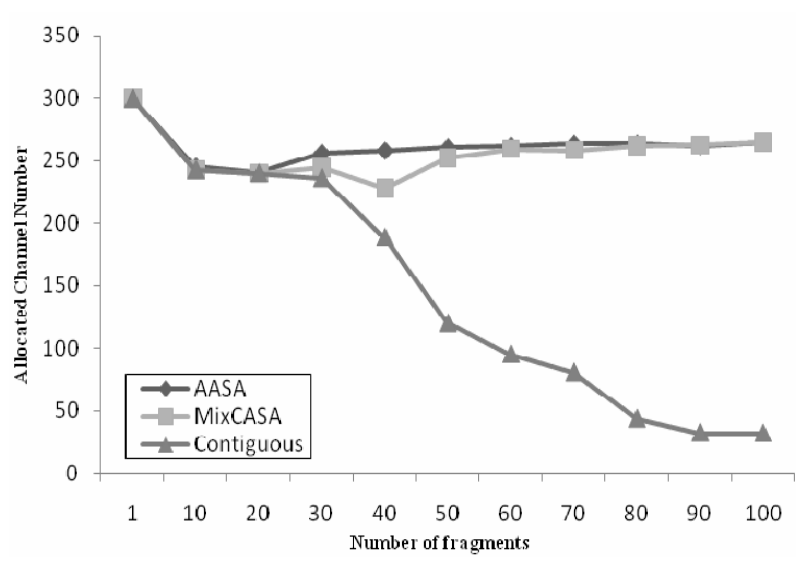

Figure 2. Number of channels allocated of different algorithms. 


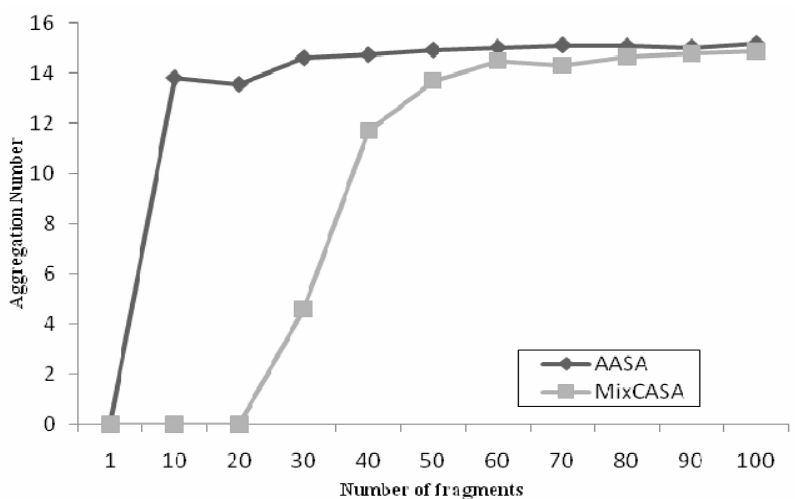

Figure 3. Aggregation number of AASA and MixCASA.

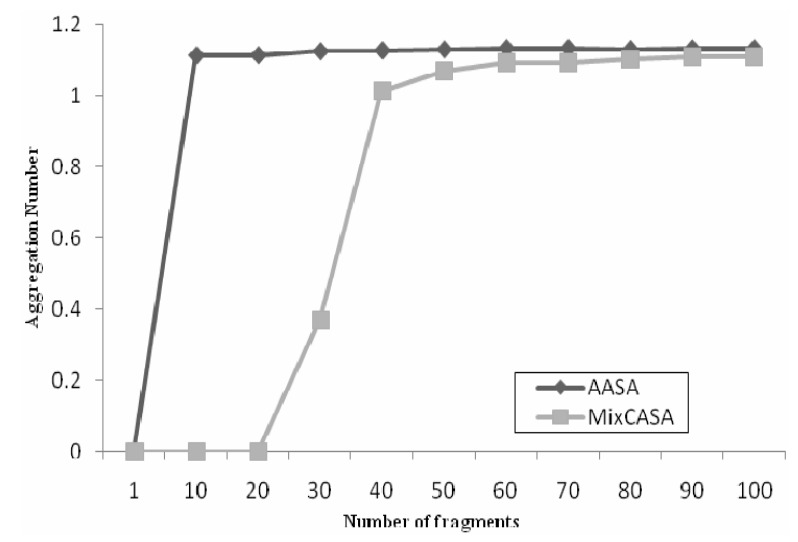

Figure 4. Converted overhead of aggregation.

\section{Conclusions}

A MixCASA algorithm has been proposed in this paper for CR based TD-LTE system. In this algorithm, spectrum resources are allocated contiguously in best-fit method if there are sufficiently enough contiguous vacant channels for a SU's demand. Otherwise spectrum aggregation is applied to guarantee the number of channels that can be allocated. Simulation results have shown that when the sensed spectrum is not extremely broken, this algorithm can guarantee a high number of allocated channels and reduce the overhead brought by excessive spectrum aggregation as well.

Because spectrum efficiency and overhead are the main factors that we try to balance, we don't take the interference SUs bring to PUs into consideration in this paper. Our future work will focus on both spectrum efficiency and power optimization.

\section{Acknowledgements}

This work is supported in part by National Key Technology R\&D Program of China (2012ZX03003006), NSFC (Grant 61271181), and National 973 Program of China (2012CB316005).

\section{REFERENCES}

[1] “LTE: The UMTS Long Term Evolution,” New York: John Wiley \& Sons, 2009.

[2] I. F. Akyildiz, W. Y. Lee, M. C. Vuran, et al., "Next Generation/Dynamic Spectrum Access/cognitive Radio Wireless Networks: A Survey,” Computer Networks, Vol. 50, No. 13, 2006, pp. 2127-2159. doi:10.1016/j.comnet.2006.05.001

[3] I. F. Akyildiz, W. Y. Lee, M. C. Vuran, et al., "A Survey on Spectrum Management in Cognitive Radio Networks," Communications Magazine, IEEE, 2008, pp. 40-48.

[4] L. Chen, W. Chen, X. Zhang, et al., "Analysis and Simulation for Spectrum Aggregation in LTE-advanced System," Vehicular Technology Conference Fall (VTC 2009-Fall), 2009 IEEE 70th. IEEE, 2009, pp. 1-6.

[5] Z. Shen, A. Papasakellariou, J. Montojo, et al., "Overview of 3GPP LTE-advanced Carrier Aggregation for 4G Wireless Communications,” Communications Magazine, IEEE, Vol. 50, No. 2, 2012, pp. 122-130. doi:10.1109/MCOM.2012.6146491

[6] M. Masmano, I. Ripoll, P. Balbastre, et al., "A Constant-time Dynamic Storage Allocator for Real-time Systems,” Real-Time Systems, Vol. 40, No. 2, 2008, pp. 149-179. doi:10.1007/s11241-008-9052-7

[7] M. Hamid, A. Mohammed and Z. Yang, "On Spectrum Sharing and Dynamic Spectrum Allocation: MAC Layer Spectrum Sensing in Cognitive Radio Networks," Communications and Mobile Computing (CMC), 2010 International Conference on IEEE, 2010, Vol. 2, pp. 183-187.

[8] M. Lee and T. J. Lee, "Multichannel MAC Protocol with Discontiguous-OFDM for Cognitive Radio Networks," Informatics Engineering and Information Science, Springer Berlin Heidelberg, 2011, pp. 594-603. doi:10.1007/978-3-642-25462-8_53

[9] Chen Y S, Liao S H. "Spectrum-aware routing in discontinuous orthogonal frequency division multiplexing-based cognitive radio ad hoc networks," Networks, IET, Vol. 1, No. 1, 2012, pp. 20-33. doi:10.1049/iet-net.2011.0002

[10] Poston J D, Horne W D. “Discontiguous OFDM Considerations for Dynamic Spectrum Access in Idle TV Channels," New Frontiers in Dynamic Spectrum Access Networks, DySPAN, First IEEE International Symposium on. IEEE, 2005, pp. 607-610.

[11] Y. Li, L. Zhao, C. Wang, et al., "Aggregation-based Spectrum Allocation Algorithm in Cognitive Radio Networks," Network Operations and Management Symposium (NOMS), IEEE, 2012, pp. 506-509.

[12] F. Huang, W. Wang, H. Luo, et al., "Prediction-Based Spectrum Aggregation with Hardware Limitation in Cognitive Radio Networks," Vehicular Technology Conference (VTC 2010-Spring), 2010 IEEE 71st., 2010, pp. 1-5.

[13] D. Chen, Q. Zhang and W. Jia, "Aggregation Aware Spectrum Assignment in Cognitive ad-hoc Networks," Cognitive Radio Oriented Wireless Networks and Communications, 2008. CrownCom 2008. 3rd International Conference on. IEEE, 2008, pp. 1-6. 